\title{
Traffic Sign Recognition System for Roadside Images in Poor Condition
}

\author{
Thongchai Surinwarangkoon, Supot Nitsuwat, and Elvin J. Moore
}

\begin{abstract}
Traffic sign detection and recognition is a difficult task, especially if we aim at detecting and recognizing signs in images captured under poor conditions. Complex backgrounds, obstructing objects, inappropriate distance of signs, shadow, and other lighting-related problems may make it difficult to detect and recognize signs in both rural and urban areas. In this paper we propose and test a system that employs image pre-processing, color filtering, color segmentation for traffic sign detection at the detection stage, feature extraction and trained neural networks for unique identification of signs at the recognition stage. The traffic sign detection and recognition system has been tested on actual roadside images taken under poor conditions. The images were selected in order to test the efficiency of the system under challenging conditions of inappropriate distance, traffic sign size, poor lighting and complex background. Suggestions are made for improving the performance of the system.
\end{abstract}

Index Terms-Image pre-processing, feature extraction, traffic sign detection and recognition, poor conditions.

\section{INTRODUCTION}

Traffic signs are an essential feature in giving important safety information to drivers about road conditions. They describe the current traffic situation, define driving directions, provide warnings about potential risks, and permit or prohibit roadway access. Automatic traffic sign recognition can help to keep a driver aware of the traffic situation and surrounding environment by seeing and highlighting signs that are ahead and/or have just been passed. A traffic sign recognition system can reduce driver's cognitive load and enhance driving safety. In addition, there are now attempts being made to develop autonomous or intelligent transportation systems. For these systems, automatic traffic sign recognition will be essential.

In a majority of published work a two-stage sequential approach involving a detection stage and a recognition (or classification) stage has been adopted for identifying traffic signs from roadside images. The aim of the detection stage is to locate a region of interest in an image and to verify that the region of interest contains a traffic sign. The aim of the recognition stage is to uniquely identify the traffic sign detected in the detection stage.

To detect possible sign candidates, color information has typically been primarily used [1]-[3] followed by geometrical

Manuscript received November 15, 2012; revised January 15, 2013.

T. Surinwarangkoon is with the Department of Business Computer, Faculty of Management Science, Suan Sunandha Rajabhat University, Bangkok, Thailand (e-mail: thongchaisurin@gmail.com).

S. Nitsuwat and E. J. Moore are with the Department of Mathematics, King Mongkut's University of Technology North Bangkok, Bangkok, Thailand (e-mail: sns@kmutnb.ac.th, ejm@kmutnb.ac.th). edge [1], [3]-[5] or corner analysis [2]. However, some authors preferred a strictly colorless approach as they did not consider color segmentation completely reliable due to its sensitivity to various factors, such as distance from the target sign, weather conditions, time of day, or reflectance of the sign's surface.

Among the studies in which color information was used for detection [1]-[3], the majority were based on non-RGB color spaces. The Hue-Saturation-Value (HSV) color model has been the most popular one as it is based on human color perception. Additionally, it is considered largely invariant to illumination changes. An HSV model has also been used in [6] to classify test sign images into several distinctive categories. An alternative method of color-detection has been based on image segmentation using the CIECAM97 color model. An interesting and robust approach to color-based detection and segmentation of road signs using IHLS color space has also been proposed [7]. In the above studies, color appearance models were adopted which are reasonable choices compared to the standard RGB model.

At the recognition stage, a pixel-based approach has often been applied and the class of the detected sign has been determined by a cross-correlation template matching technique [1], [8]. A feature-based approach has also frequently been used with up to $95 \%$ success recognition rate being reported in experiments involving still camera images. However, the majority of previous studies have focused only on narrow subsets of traffic signs or traffic situations. Typically, authors have only tested their models on a single semantic category, e.g. speed limit signs, or relatively dissimilar signs from multiple categories.

Detection and recognition of traffic signs is very challenging in the real world environment. Some of the difficulties are as follows. Images of the same traffic sign can have different qualities when taken under various conditions. A traffic sign image taken from a long distance has a small size that may provide inadequate information for detection and classification. A large size image of a sign can provide good quality information but it may require excessively long processing time. The color of a roadside image may be distorted if it is taken under light conditions that are too dark or too bright. Bad weather conditions are also likely to have a detrimental effect on the quality of the images. Complex backgrounds are also likely to cause difficulties at the detection stage because of the possible presence of background objects similar in size, color and shape to a traffic sign.

In this work, we report experiments on traffic sign detection and recognition for roadside images in poor conditions. In particular, we examine effects of distance, size of traffic sign, light conditions, and complex background. 
The effect of the poor conditions on the overall traffic sign recognition accuracy is shown and the performances of the classifier for various conditions of roadside images are compared. The experiments were conducted on relatively large size databases of Thai traffic signs containing 33 red prohibitory signs, 19 blue prohibitory signs, 53 general warning signs (yellow) and 24 construction area warning signs (amber).

\section{TRAFFic Sign Detection And Recognition System \\ A. Traffic Signs}

Because traffic signs provide important information for drivers about road condition and hazards, their shapes and colors are selected by traffic authorities to make them easily recognizable by humans. In Thailand, traffic signs are divided into three main classes: prohibitory signs (red and blue signs), general warning signs (yellow signs), and warning signs at construction areas (amber signs). Examples of traffic signs in Thailand are shown in Table I.

\section{TABLE I: EXAMPLES OF TRAFFIC SIGNS IN THAILAND}

Traffic Sign Types
Example 1
Prohibitory signs
(Red signs)
$\begin{aligned} & \text { Example 2 } \\ & \text { (Blue signs) }\end{aligned}$
$\begin{aligned} & \text { General warning signs } \\ & \text { (Yellow signs) }\end{aligned}$
Warning signs
at construction areas
(Amber signs)

\section{B. Overview of Traffic Sign Detection and Recognition} System

Fig. 1 shows an overview of the proposed system. The main stages of our implementation are: image pre-processing, color filtering, color segmentation and traffic sign detection, feature extraction, and traffic sign recognition. We describe each step in detail in the following sections.

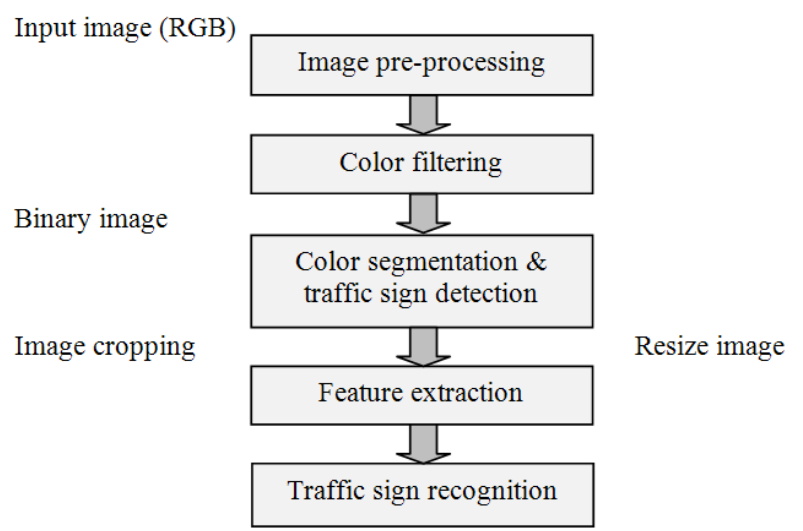

Fig. 1. Stages of traffic sign detection and recognition system.

\section{TRAFFIC Sign DETECTION}

\section{A. Image Pre-Processing}

In this paper, images of traffic signs have been taken in Thailand under a variety of environmental conditions using a digital camera (Kodak M200). These traffic sign images were preprocessed to find appropriate ranges of distance, normalized standard size, and colors. A standard RGB color model was used for measuring traffic sign colors under various viewing conditions. This model can estimate color appearance as accurately as human perception. Table II shows RGB values of colors usually used in Thai traffic signs, namely black, blue, red, yellow, amber, and white.

TABLE II: RGB VALUES FOR EACH COLOR IN TRAFFIC SIGNS

\begin{tabular}{cccc}
\hline \hline \multirow{2}{*}{ Color } & \multicolumn{3}{c}{ RGB Value } \\
& $\mathrm{R}$ & $\mathrm{G}$ & $\mathrm{B}$ \\
\hline Black & 0 & 0 & 0 \\
Blue & 0 & 0 & 255 \\
Red & 255 & 0 & 0 \\
Yellow & 255 & 255 & 0 \\
Amber & 150 & 70 & 0 \\
White & 255 & 255 & 255 \\
\hline \hline
\end{tabular}

The sign images input into the experiments had a variety of distances, traffic sign sizes, light conditions and backgrounds as shown below.

1) Roadside images for distance testing were detected at a physical distance from the camera of approximately 10-30, 31-50, 51-70, and 71-90 meters (Fig. 2).

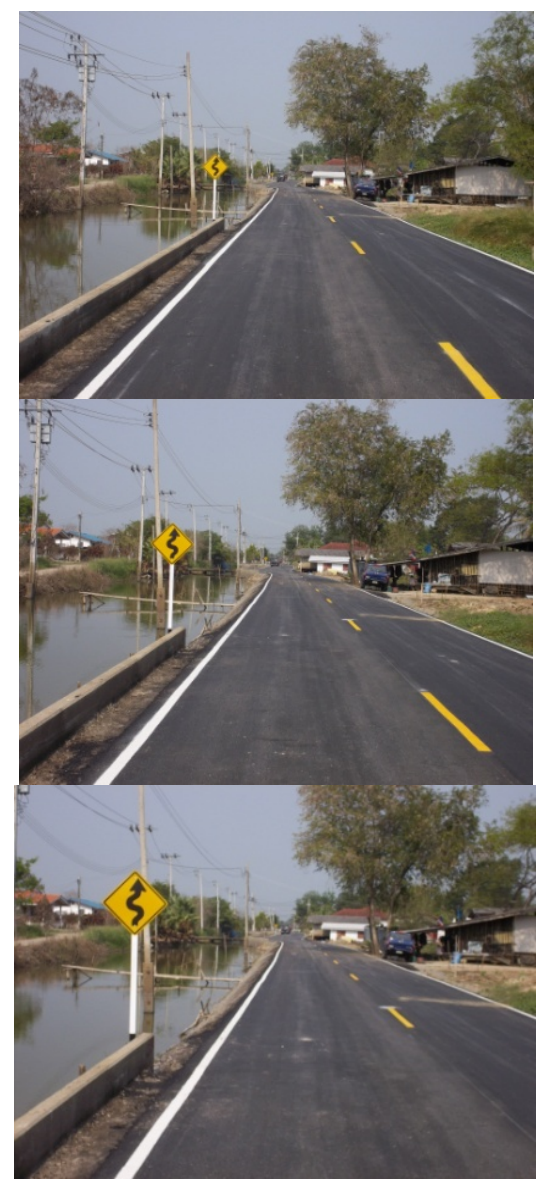

Fig. 2. Examples of traffic sign images at different distances from camera; 51-70 m (top), 31-50 m (middle), and 10-30 m (bottom). 
2) The sizes of the test traffic signs in both the standard database and in roadside images were normalized to $70 \times$ $70,100 \times 100,130 \times 130$, and $160 \times 160$ pixels for size testing of signs.

3) Roadside images (Fig. 3) for testing light conditions were taken in shady, normal and bright light conditions by a digital camera (Kodak M200) which has limited ability to adjust its sensitivity to prevailing light conditions.

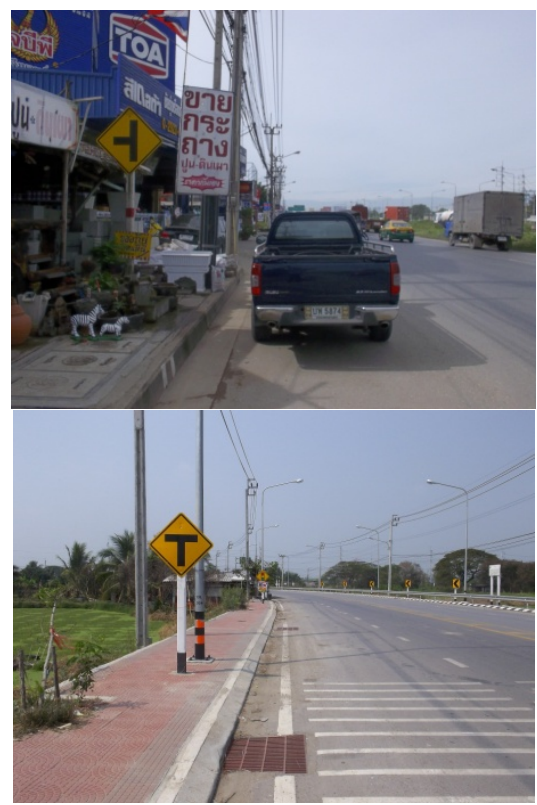

Fig. 3. Example of roadside images in shady conditions (top) and bright conditions (bottom).

4) A group of different background images were collected from urban and rural areas for testing (Fig. 4).

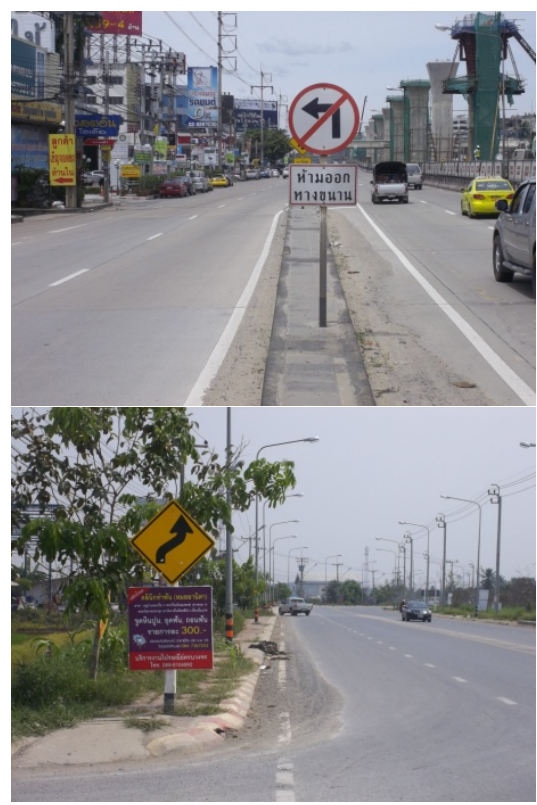

Fig. 4. Examples of complex background roadside images from an urban area (top) and a rural area (bottom).

After a roadside image was prepared, it was input into the color filtering stage.

\section{B. Color Filtering}

At this stage, red, green and blue values for each pixel of a roadside image were captured. Then images from red, blue, yellow and amber filtering were transformed to binary mode.

\section{Color Segmentation and Traffic Sign Detection}

Color segmentation was then applied to the filtered images in binary mode to find the contour of the traffic sign.

Based on the range of sign colors, regions containing traffic signs were segmented from the rest of the images. Sometimes, other objects, such as warning red line bars beside the road surface or blue advertisement signs were also segmented. However, these non-traffic sign segments could be rejected during the detection stage. If the size of the segment was too small or not a traffic sign, then the non-traffic sign segments were discarded. Therefore, a traffic sign was detected by this process. Fig. 5 shows an example of an image after the color segmentation and traffic sign detection stage.

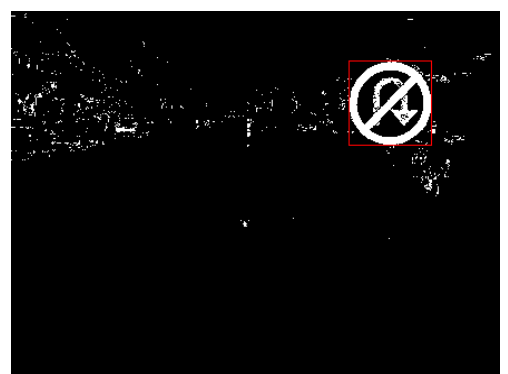

Fig. 5. An example of traffic sign detected by color segmentation.

\section{FEATURE EXTRACTION}

At this stage, an image of the traffic sign was cropped and resized in order to match with images in a standard test library. Fig. 6 shows an example of cropped traffic sign image. It is resized to $70 \times 70$ pixels. Then, $70 \times 70$ pixel image is divided into $7 \times 7$ grids. Thus, the image is divided into 49 nodes, each node consists of 10x10 pixels as shown in Fig. 7.

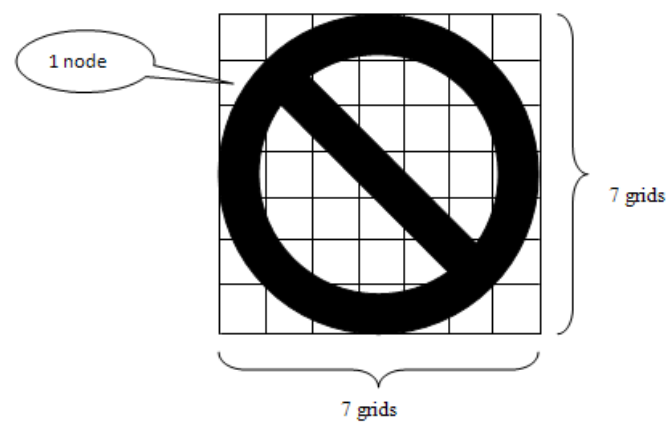

Fig. 6. $70 \times 70$ pixel image divided into $7 \times 7$ grids (Total $=49$ nodes $)$.

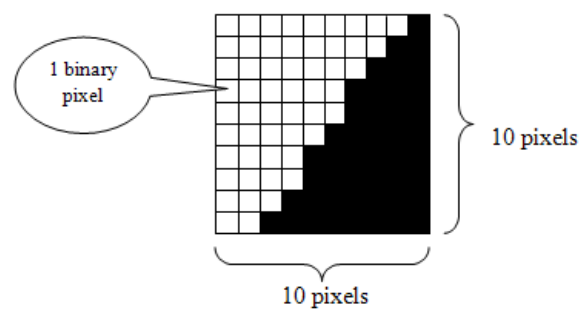

Fig. 7. Diagram of a node.

The average binary value of each node is calculated using (1).

Average binary value $=$ Sum of binary value

$\div$ Total amount of pixels. 
After that, the image information is then sent to traffic sign feature database.

\section{TRAFFIC SIGN RECOGNITION}

Traffic sign recognition requires a classification technique. As reported previously [9]-[11], we have found that Neural Networks can be trained to be effective classifiers of traffic signs.

There are many neural network models, but the basic structure involves a system of layered, interconnected nodes. The nodes are arranged to form an input layer, hidden layer, and output layer, with nodes in each layer connected to all nodes in neighboring layers.

Information enters the network at the input layer nodes and moves along weighted links to nodes in the hidden and output layers. Each node combines information from all nodes in the previous layer, resulting in a final output.

Complexities in the data are captured through the number of nodes in the hidden layer. The weights are determined by iteration to produce the lowest error in the output. To avoid overfitting to the data, a neural network is usually trained on a subset of inputs and outputs to determine weights and subsequently validated on the remaining data to measure the accuracy of predictions. In this paper, neural networks are used in classification and recognition of traffic signs.

In our experiments, four neural network modules were trained and tested for identifying signs in each of the red, blue, yellow and amber groups shown in Table I. An overview of the training and testing stages of each neural network module are shown in Fig. 8.
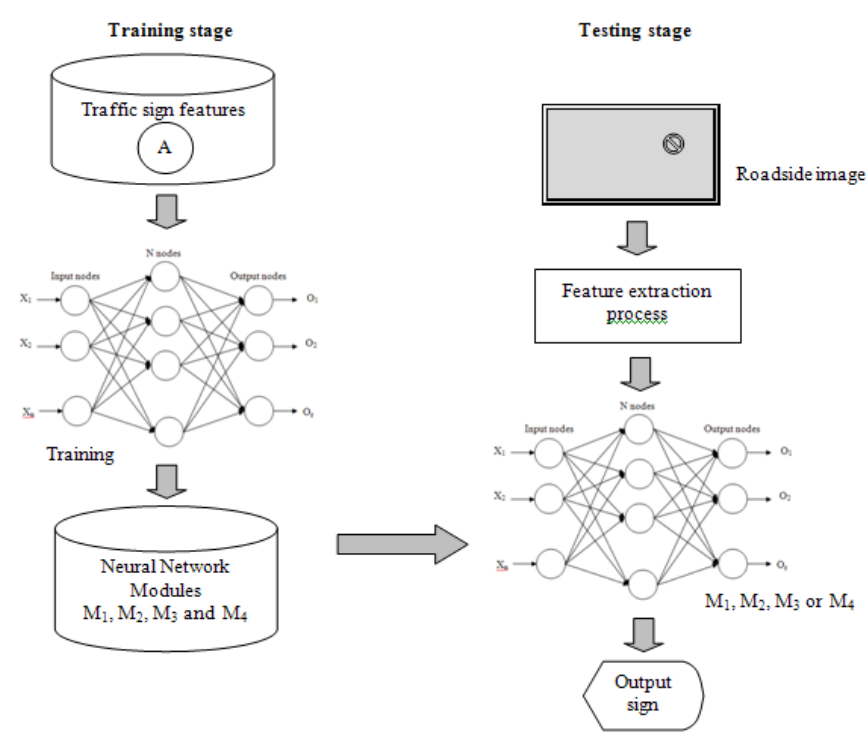

Fig. 8. Overview of training stage and testing stage of neural network modules.

At the training stage, a neural network module for a color group was trained to recognize each of the different signs in that color group. Then, the training weights generated for each color group were stored in neural network modules $\left(\mathrm{M}_{1}\right.$, $\mathrm{M}_{2}, \mathrm{M}_{3}$ and $\mathrm{M}_{4}$ for red, blue, yellow and amber groups).

At this step, each group has 49 input nodes in the network but a different number of output nodes; 33 output nodes for red, 19 for blue, 53 for yellow and 24 for amber.
Table III illustrates the training image quantities for each traffic sign types. A total of 1,032 traffic sign images were input at the training stage.

\begin{tabular}{cc} 
TABLE III: TRAINING IMAGE QUANTITIES FOR EACH TRAFFIC SIGN TYPE \\
\hline \hline Traffic Sign Types & Number of Training Images \\
\hline Prohibitory signs (Red signs) & 264 \\
Prohibitory signs (Blue signs) & 152 \\
General warning signs (Yellow signs) & 424 \\
Warning signs at construction areas & 192 \\
Total & 1,032 \\
\hline \hline
\end{tabular}

At the testing stage, a roadside image was input into the system. After the traffic sign had been detected, it was then sent to the appropriate neural network color module for identification.

\section{EXPERIMENTAL RESULTS}

A group of roadside images was input to the traffic sign detection and recognition system in order to analyze its performance for roadside images in poor conditions in terms of distance, size of traffic sign, light condition, and complex background. The experimental results are shown in the following subsections.

\section{A. Distance Testing Result}

Table IV illustrates the test results of traffic sign detection and recognition for roadside images from different distances.

\begin{tabular}{cccccc}
\multicolumn{2}{c}{ TABLE IV: DISTANCE TEST RESULTS } \\
\hline \hline $\begin{array}{c}\text { Sign } \\
\text { Distances } \\
\text { (meter) }\end{array}$ & $\begin{array}{c}\text { Number } \\
\text { of Inputs }\end{array}$ & \multicolumn{2}{c}{ Detection } & \multicolumn{2}{c}{ Recognition } \\
Number & $(\%)$ & $\begin{array}{c}\text { Number } \\
\text { Correct }\end{array}$ & $\begin{array}{c}(\%) \\
\text { Correct }\end{array}$ & Correct & Correct \\
\hline $10-30$ & 20 & 20 & 100 & 20 & 100 \\
$31-50$ & 20 & 19 & 95 & 19 & 100 \\
$51-70$ & 20 & 18 & 90 & 17 & 94 \\
$71-90$ & 20 & 15 & 75 & 12 & 80 \\
\hline \hline
\end{tabular}

From the results shown in Table IV, it can be seen that detection of a traffic sign at 10-30 meters distance has an accuracy of $100 \%$ and recognition has an accuracy of $100 \%$. It can also be seen that the detection and recognition accuracies decrease as the distance increases, with a $75 \%$ detection accuracy and an $80 \%$ recognition accuracy at $71-90$ meters.

When a traffic sign image is taken from a long distance, it is more difficult for the detection stage to judge that it is a traffic sign as there may be other similar objects along the roadside. Moreover, the cropped image of a sign detected from a long distance will be smaller and be more blurred after resizing at feature extraction stage. The signs at 71-90 meters have low detection and recognition accuracy because the input information of images taken from this distance is low quality.

\section{B. Traffic Sign Size Testing}

Table V shows testing results of traffic sign detection and 
recognition for different sizes of normalized traffic sign images in both the standard database and in roadside images. In this test, all roadside images were taken at approximately 10-30 meters from camera. In the test roadside images used in this comparison, the color filtering stage gave a clear detection and classification of the color type of the traffic sign image. The processing time results were obtained on a $3.1-\mathrm{GHz}$ Intel Core i5 with frame dimensions of $720 \times 576$ pixels.

Base on the results in Table $\mathrm{V}$, traffic signs can be recognized at $100 \%$ accuracy when test signs in both the standard database and in roadside images were normalized to 100x100, 130x130, and 160x160 pixels.

TABLE V: TRAFFiC Sign SIZE TEST RESUlts

\begin{tabular}{ccccc}
\multicolumn{5}{c}{ TABLE V: TRAFFIC SIGN SIZE TEST RESULTS } \\
\hline $\begin{array}{c}\text { Sign Sizes } \\
\text { (pixel) }\end{array}$ & $\begin{array}{c}\text { Number of } \\
\text { Inputs }\end{array}$ & $\begin{array}{c}\text { Recognition } \\
\text { Number } \\
\text { Correct }\end{array}$ & $\begin{array}{c}\text { Average } \\
\text { Correct }\end{array}$ & $\begin{array}{c}\text { Processing Time } \\
\text { (second) }\end{array}$ \\
\hline $70 \times 70$ & 30 & 29 & 96.5 & 0.273 \\
$100 \times 100$ & 30 & 30 & 100 & 0.289 \\
$130 \times 130$ & 30 & 30 & 100 & 0.325 \\
$160 \times 160$ & 30 & 30 & 100 & 0.356 \\
\hline \hline
\end{tabular}

It is clear that traffic sign recognition for the smaller sizes of normalized traffic sign images requires less computation time than larger sizes.

These results show that traffic sign recognition for 100x100 pixel images has high accuracy, and that it also requires a low computational time. Moreover, we found that off-line Neural Network training for the smaller sizes of normalized traffic sign images can run faster than for the larger sizes.

\section{Light Condition Testing}

Table VI shows the test results of traffic sign detection and recognition for roadside images in various light conditions.

The results show that the system correctly detected 17 of 30 traffic signs from roadside images taken in shady conditions, 23 of 30 traffic signs taken in bright conditions, and 30 of 30 taken in normal conditions. The system correctly recognized $88 \%$ of detected traffic signs taken in shady conditions, $91 \%$ of detected traffic signs taken in bright condition, and $100 \%$ of detected signs taken in normal light condition.

TABLE VI: LIGHT CONDITION TEST RESULTS

\begin{tabular}{cccccc}
\hline \hline & \multicolumn{2}{c}{ TABLE VI: LIGHT CONDITION TEST RESULTS } \\
$\begin{array}{c}\text { Light } \\
\text { Conditions }\end{array}$ & $\begin{array}{c}\text { Number } \\
\text { of Inputs }\end{array}$ & $\begin{array}{c}\text { Detection } \\
\text { Number } \\
\text { Correct }\end{array}$ & $\begin{array}{c}\text { Recognition } \\
\text { Correct }\end{array}$ & $\begin{array}{c}\text { Number } \\
\text { Correct }\end{array}$ & $\begin{array}{c}(\%) \\
\text { Correct }\end{array}$ \\
\hline Shady & 30 & 17 & 57 & 15 & 88 \\
Normal & 30 & 30 & 100 & 30 & 100 \\
Bright & 30 & 23 & 77 & 21 & 91 \\
\hline \hline
\end{tabular}

Some roadside images taken in bright conditions which the system could not detect traffic sign initially could be detected after a pre-processing stage. However, it was found to be difficult to improve detection of traffic signs from roadside image taken in shady condition by the pre-processing methods that we used. We found that the light adjustment did not change the traffic sign colors which remained similar to the colors in the original shady condition image.

\section{Background Testing}

Table VII illustrates test results of traffic sign detection and recognition for roadside images with complex backgrounds from different locations.

TABLE VII: BACKGROUND TEST RESULTS

\begin{tabular}{cccccc}
\multicolumn{1}{c}{ TABLE VII: BACKGROUND TEST RESULTS } \\
\hline $\begin{array}{c}\text { Traffic Sign } \\
\text { Locations }\end{array}$ & $\begin{array}{c}\text { Number } \\
\text { of Inputs }\end{array}$ & $\begin{array}{c}\text { Dumber } \\
\text { Correct }\end{array}$ & $\begin{array}{c}(\%) \\
\text { Correct }\end{array}$ & $\begin{array}{c}\text { Number } \\
\text { Correct }\end{array}$ & $\begin{array}{c}\text { Recognition } \\
\text { Correct }\end{array}$ \\
\hline Urban areas & 30 & 28 & 93 & 27 & 96 \\
Rural areas & 30 & 29 & 97 & 28 & 97 \\
Total & 60 & 57 & 95 & 55 & 96.5 \\
\hline \hline
\end{tabular}

From the results shown in Table VII, it can be seen that the system correctly detected $93 \%$ of traffic signs from roadside images in urban areas, $97 \%$ in rural areas, and approximately $95 \%$ for both areas. The system correctly recognized $96 \%$ of traffic signs in urban areas, $97 \%$ in rural areas, and $96.5 \%$ for both areas. In general, roadside images in urban areas have more complex background than roadside images in rural areas because urban areas contain colorful advertisement signs, company signs, and other non-traffic signs which can have similar characteristics to traffic signs. However, overall the system showed reasonable performance when tested with challenging images containing complex backgrounds. Sometime, there was more than one traffic sign within a roadside image. We found that these signs were correctly detected and recognized by the proposed system.

\section{CONCLUSION}

In this paper, experimental results of traffic sign detection and recognition for roadside images in various conditions have been discussed in terms of sign distance, size of traffic sign, light condition, and complex background. Traffic signs at 10-30 meters distance were found to be at an appropriate distance for high accuracy traffic sign detection and recognition, whereas at longer distances lower accuracies of detection and recognition rate were obtained. Traffic sign recognition for $100 \times 100$ pixels images was found to have high accuracy of recognition rate and a fast computational time. We found that the quality of some roadside images in bright condition could be sufficiently improved by light adjustment at pre-processing stage to permit correct detection and recognition. Finally, the proposed traffic sign detection and recognition system was found to offer satisfactory performance when tested on images from both rural and urban areas with challenging complex backgrounds.

Future work will mainly focus on improving the efficiency of the traffic sign detection stage in order to decrease noise and increase quality of input roadside images. We will also analyze performance of detection and recognition for physically damaged and obstructed real world images of traffic signs.

Moreover, we will apply ontology-based knowledge in order to improve traffic sign recognition, guide image interpretation and to give more detailed descriptions of traffic 
conditions and improved recommendations in a driver assistance system.

\section{ACKNOWLEDGMENT}

Thongchai Surinwarangkoon thanks Assistant Professor Dr. Supot Nitsuwat and Dr. Elvin J. Moore for their kind support. Financial assistance for Thongchai Surinwarangkoon was provided by a scholarship from Suan Sunandha Rajabhat University, Bangkok, Thailand.

\section{REFERENCES}

[1] G. Piccioli, E. De Micheli, P. Parodi, and M. Campani, "A robust method for road sign detection and recognition," Image and Vision Computing, vol. 14, pp. 209-223, 1996.

[2] A. de la Escalera, L. E. Moreno, M. A. Salichs, and J. M. Armingol, "Road traffic sign detection and classification," IEEE Trans. on Industrial Electronics, vol. 44, pp. 848-859, 1997.

[3] X. W. Gao, L. Podladchikova, D. Shaposhnikov, K. Hong, and N. Shevtsova, "Recognition of traffic signs based on their colour and shape features extracted using human vision models," Journal of Visual Communication and Image Representation, vol. 17, pp. $675-685,2006$

[4] G. Loy, N. Barnes, D. Shaw, and A. Robles-Kelly, "Regular polygon detection," in Proc. 10th IEEE International Conf. on Computer Vision, 2005 , pp. 8-16.

[5] M. A. Garcia-Garrido, M. A. Sotelo, and E. Martin-Gorostiza, "Fast traffic sign detection under changing lighting conditions," in Proc. IEEE International Conf. on Intelligent Transportation Systems, 2006, pp. 811-816.

[6] P. Paclik, J. Novovicova, P. Pudil, and P. Somol, "Road signs classification using the Laplace kernel classifier," Pattern Recognition Letters, vol. 21, pp. 1165-1173, 2000.

[7] H. Fleyeh, "Color detection and segmentation for road and traffic signs," in Proc. IEEE Conf. on Cybernetics and Intelligent Systems, Singapore, 2004, pp. 8-16.

[8] A. de la Escada, J. M. Armingol, J. M. Pastor, and F. J. Rodriguez, "Visual sign information extraction and identification by deformable models for intelligent vehicles," IEEE Trans. on Intelligent Transportation Systems, pp. 57-68, 2004.

[9] T. Surinwarangkoon, S. Nitsuwat, and E. J. Moore, "Traffic sign recognition by color segmentation and neural network," in Proc. 4th International Conf. on Machine Vision, Singapore, 2012, pp. 330-335.

[10] T. Surinwarangkoon, S. Nitsuwat, and E. J. Moore, "Traffic sign recognition by color filtering and particle swarm optimization," in Proc. 4th International Conf. on Computer Research and Development Chengdu, 2012, pp. 55-59.

[11] T. Surinwarangkoon, S. Nitsuwat, and E. J. Moore, "Comparing Classification Performances between Neural Networks and Particle Swarm Optimization for Traffic sign recognition," in Proc. 5th WSEAS International Conf. on Visualization, Imaging and Simulation, Malta, 2012, pp. 19-23.

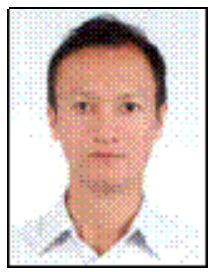

Thongchai Surinwarangkoon was born in Suratthani, Thailand in 1972. He obtained B.Sc. degree in mathematics from Chiang Mai University, Thailand in 1995 and M.Sc. degree in management of information technology from Walailak University, Thailand in 2005.

He is now a Lecturer in Department of Business Computer, Suan Sunandha Rajabhat University, Bangkok, Thailand. His research interests include digital image processing, knowledge-based system and information technology application in business.

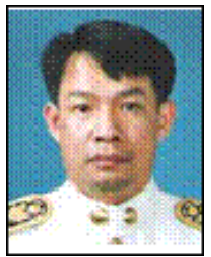

Supot Nitsuwat obtained B.Sc. degree in Mathematics from Ramkhamhaeng University, Bangkok, Thailand in 1981 and M.Sc. degree in applied mathematics from Mahidol University, Bangkok, Thailand in 1985. He obtained a Ph.D. degree in computer science from the University of New South Wales, Australia in 2001.

$\mathrm{He}$ is currently with the King Mongkut's University of Technology North Bangkok, where he has been an Assistant Professor with Mathematics Department. His research interests include digital image processing, pattern recognition, fuzzy sets and systems.

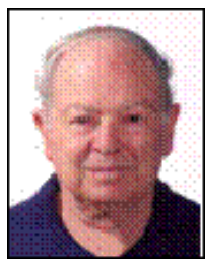

Elvin J. Moore was born in Western Australia in 1936. He obtained B.Sc. and M.Sc. in physics from the University of Western Australia in 1958 and 1959 respectively. He obtained a Ph.D. degree in theoretical physics from Harvard University, Cambridge, MA, USA in 1966.

From 1966 to 1998 he was in the Department of Applied Mathematics, University of New South Wales. From 1998 to the present, he has been a Foreign Lecturer in the Department of Mathematics, King Mongkut's University of Technology North Bangkok, Thailand. His present research interests are mainly in areas of numerical analysis and mathematical modeling of diseases and epidemics, marine systems, traffic flow using methods from differential equations, time-delay differential equations, difference equations, graph theory and autormata theory. 